\title{
Reentrant wetting transition in the spreading of cellular aggregates $\dagger$
}

\author{
Grégory Beaune, (ID $\ddagger^{\mathrm{ab}}$ Guillaume Duclos, (D) $\ddagger^{\mathrm{ab}}$ Nada Khalifat, $\ddagger^{\mathrm{ab}}$ \\ Tomita Vasilica Stirbat, ${ }^{a b}$ Danijela Matic Vignjevic ${ }^{c d}$ and \\ Françoise Brochard-Wyart (DD *ab
}

\begin{abstract}
We study spreading on soft substrates of cellular aggregates using CT26 cells that produce an extracellular matrix (ECM). Compared to our previous work on the spreading of S180 cellular aggregates, which did not secrete ECMs, we found that the spreading velocity of the precursor film is also maximal for intermediate rigidities, but new striking features show up. First, we observed a cascade of liquidgas-liquid $(L / G / L)$ transitions of the precursor film as the substrate rigidity is decreased. We attribute the $L / G$ transition to a decrease of cell/cell adhesion resulting from the weakening of the cell/substrate adhesion. We attribute the reentrant liquid phase $(G / L)$ observed on soft substrates to the slow spreading of the aggregates on ultra-soft substrates, which gives time to the cells to secrete more ECM proteins and stick together. Second, a nematic order appears in the cohesive (liquid) states of the precursor film, attributed to the gradient of cell's velocities.
\end{abstract}

\section{Introduction}

Our aim is to study the spreading of cells secreting an extracellular matrix on rigid and soft substrates. During morphogenesis and cancer development, cell migration, tumor growth, and metastasis are determined, in part, by cell-cell interactions mediated by the extracellular matrix (ECM). The ECM has many functions, including acting as a physical scaffold, facilitating interactions between different cell types, and providing survival and differentiation signals. Cells sense, analyze and integrate both chemical and physical external cues from their environment, and subsequently change their morphology and migrating behaviors. ${ }^{1,2}$ Previously, a maximal cell speed was observed

\footnotetext{
${ }^{a}$ UPMC Univ Paris 06, UMR 168, Institut Curie, 26 rue d'Ulm, 75248 Paris Cedex 05, France. E-mail: brochard@curie.fr

${ }^{b}$ CNRS, UMR 168, Institut Curie, 26 rue d'Ulm, 75248 Paris Cedex 05, France

${ }^{c}$ UPMC Univ Paris 06, UMR 144, Institut Curie, 26 rue d'Ulm,

75248 Paris Cedex 05, France

${ }^{d}$ CNRS, UMR 144, Institut Curie, 26 rue d'Ulm, 75248 Paris Cedex 05, France $\dagger$ Electronic supplementary information (ESI) available: Fig. S1. Schematic representation of a spreading aggregate. Fig. S2. Confocal pictures of stained aggregates on gels. Fig. S3. The effect of the flow on the alignment of CT26 cells monolayer on glass. Fig. S4. Wound healing experiment. Fig. S4. S180 transfected cell aggregate spreading on glass. Movie 1. CT26 aggregate spreading on glass. Movie 2. CT26 aggregate spreading on $16.4 \mathrm{kPa}$ PAA. Movie 3. CT26 aggregate spreading on $10.6 \mathrm{kPa}$ PAA. Movie 4. CT26 aggregate spreading on $7.4 \mathrm{kPa}$ PAA. Movie 5. CT26 aggregate spreading on $2.8 \mathrm{kPa}$ PAA. Movie 6. CT26 aggregate spreading on $1 \mathrm{kPa}$ PAA. Movie 7. CT26 aggregate spreading on a glass substrate observed using 2-photon microscopy. See DOI: 10.1039/c7sm00768j

\$ These authors contributed equally to the manuscript.
}

on a $20 \mathrm{kPa}$ substrate for vascular smooth muscle cells (SMCs) cultured on substrates presenting a high ECM ligand density. ${ }^{3}$ Depending on the cell type and tissue environment, cells can migrate in two major ways: individually, when cell-cell junctions are absent or collectively as multicellular groups, where cells remain cohesive and cell-cell adhesion is retained. ${ }^{4}$ This multicellular mode, or collective migration, is commonly used by carcinomas. ${ }^{5}$ Preventing the invasion of tumors represents the main medical challenge. Thus, it is crucial to understand the bio-chemical and physical parameters that control the spreading of multicellular systems. To successfully investigate the pathobiology of human cancer, it is necessary to maintain or recreate in culture the typical three-dimensional (3D) spatial conformation of the tissue. Cellular aggregates have been used in experimental cancer research for several decades and serve as in vitro models of tumors to study the mechanisms involved in proliferation and metastasis. ${ }^{6,7}$ We previously showed that aggregates behave as liquid droplets: ${ }^{8}$ they have a surface tension and are able to form spheroids to minimize their surface energy. They spread like viscous drops, where the parameters controlling the wetting properties (adhesion, viscosity and friction coefficient) depend upon cellular activity. The analogy between tissues and liquids has been very fruitful to describe the spreading of tissues, using the physics of capillarity and wetting ${ }^{7-10}$ extended to out of equilibrium systems. Stationary dissipative states have been named liquid, gas, and nematic in analogy with thermodynamics states. The spreading of tissue results from a competition between cell-cell adhesion $\left(W_{\mathrm{CC}}\right)$ controlled by cadherins and cell-substrate adhesion $\left(W_{\mathrm{CS}}\right)$ controlled by integrins binding to fibronectin or 
other ECM proteins. $W_{\mathrm{CS}}$ can be tuned either chemically using a mixture of PEG and fibronectin to coat the substrate or physically by varying substrate rigidity. ${ }^{11,12}$ The sign of the effective spreading parameter $S=W_{\mathrm{CS}}-W_{\mathrm{CC}}$ controls the wetting properties. ${ }^{13}$ If the spreading parameter $S<0$, cell-cell adhesion dominates and the aggregate does not spread, which is named "partial wetting". If the spreading parameter $S>0$, a "complete wetting" occurs, and a "precursor film" made of a cellular monolayer flows outwards from the aggregate. For large values of $W_{\mathrm{CC}}$, the precursor film is a cohesive liquid. As $W_{\mathrm{CC}}$ decreases, a transition from a liquid to a gas state is observed, where cells detach individually from the aggregate and disseminate. ${ }^{10}$ This corresponds to the epithelial-mesenchymal transition (EMT) involved in cancer metastasis and embryonic development. ${ }^{14}$ EMT marks the transition from a collective invasion pattern to single-cell invasion mechanism where cells lose their cell-cell junctions via a variety of mechanisms. ${ }^{15}$

Previously, ${ }^{7,10}$ we studied the spreading of the sarcoma 180 (S-180) cell line on glass and soft gels coated with fibronectin. These cells do not secrete an extracellular matrix and have a tunable level of cadherin expression, so $W_{\mathrm{CC}}$ can be adjusted. ${ }^{10}$ We showed that $W_{\mathrm{CC}}$ can also be reduced by decreasing cellsubstrate adhesion $W_{\mathrm{CS}}$ on soft substrates, where the focal adhesions are weaker, due to the cross-talk between the integrins and the cadherins. ${ }^{7,16}$ A wetting transition when varying the substrate rigidity from complete to partial wetting was observed for a critical value of the gel elastic modulus $E$ of order $8 \mathrm{kPa}^{11}$ We also reported a liquid-gas transition of the precursor film, when we decreased $W_{\mathrm{CC}}$ either by tuning the level of cadherins ${ }^{10}$ or the substrate rigidity. ${ }^{11}$ We showed that the liquid states observed are cohesive, and that the level of expression of cell adhesion molecule's (CAM) proteins can tune the cohesion.

Regarding the dynamics of spreading, ${ }^{7,10}$ we observed that the precursor film spreads with a diffusive law characterized by a diffusion coefficient $D$. To interpret our results, we introduced a new mechanism for tissue dynamics named permeation, which describes how cells migrate from the $3 \mathrm{D}$ aggregate into the 2D monolayer. ${ }^{7}$ One outcome of our model was that $D=$ $R_{\mathrm{a}} V^{*}$ where $R_{\mathrm{a}}$ is the radius of the aggregate and $V^{*} \sim S / \eta$ is a characteristic spreading velocity, with $\eta$, the tissue viscosity. ${ }^{17}$ On soft substrates, we found that the diffusion coefficient $D$ is not monotonic with the elastic modulus and is maximized for $E=16 \mathrm{kPa}^{11}$ Below that value, the cohesion of the film decreases and the monolayer goes from a dense "liquid" to a dilute "gas" state.

In this paper, we advance our understanding of the wetting of cellular aggregates by employing cells which secrete an extracellular matrix and are therefore a better model of in vivo tumors. ${ }^{18}$ For this purpose we use colon carcinoma CT26 cells which secrete different ECM proteins (laminin, fibronectin, collagen IV, and collagen I) as shown by immunostaining of CT26 aggregates. ${ }^{19}$ To investigate the invasive behavior of these highly aggressive cells, ${ }^{6}$ we study the spreading on glass and polyacrylamide (PAA) gels with a decreasing elastic modulus $E$. We characterize the dynamics of spreading $\left(V^{*}\right)$, the precursor film density, the cellular flow field using particle imaging velocimetry (PIV), and the cells' orientation. We find a reentrant liquid crystal-gas-liquid crystal phase transition as we decrease the substrate rigidity.

\section{Materials and methods}

\section{A. Cell culture and aggregate preparation}

We used WT mouse colon carcinoma CT26 cells (ATCC CRL2638; American Tissue Culture Collection). Cells were maintained in a Dulbecco's Modified Eagle Medium (DMEM, from Invitrogen) supplemented with $10 \%$ (vol/vol) Fetal Bovine Serum (FBS, from Invitrogen) and antibiotics $\left(100 \mu \mathrm{g} \mathrm{mL}{ }^{-1}\right.$ streptomycin and $100 \mathrm{U} \mathrm{mL}^{-1}$ penicillin, Gibco BRL) in a humidified atmosphere containing $5 \% \mathrm{CO}_{2}$ at $37{ }^{\circ} \mathrm{C}$. Cells were grown to confluent monolayers to prepare spheroids following the hanging droplet method. ${ }^{20}$ After a 3 day or 6 day incubation, the cells formed aggregates of diameter ranging from 100 to $500 \mu \mathrm{m}$.

Murine Sarcoma (S-180) cells were transfected to express E-cadherin or, the liver cell adhesion molecule (L-CAM) structurally related to $\mathrm{N}$-cadherin, at their surface. $\mathrm{S} 180$ were cultured using the same protocol as the CT26 cell line, as well as the aggregate preparation.

\section{B. Preparation of fibronectin coated substrates}

Glass substrates. Twenty-five millimeters circular glass coverslips were sonicated in ethanol for $5 \mathrm{~min}$, dried at ambient temperature, and exposed to deep UV for $10 \mathrm{~min}$. A fibronectin (Sigma-Aldrich) coating was applied using a $0.1 \mathrm{mg} \mathrm{mL}^{-1}$ solution of fibronectin in PBS solution ( $\mathrm{pH} \mathrm{7.4)}$ for $1 \mathrm{~h}$.

Soft substrates. We synthesize flexible polyacrylamide gels (PAA) by adapting a published technique. ${ }^{21}$ The substrate is prepared by allowing polyacrylamide solutions to polymerize between two chemically modified glass cover slips (Fisher Scientific, Pittsburgh, PA). A $25 \mathrm{~mm}$-diameter glass coverslip is sonicated in ethanol for $10 \mathrm{~min}$, dried at room temperature and exposed to deep UV for $5 \mathrm{~min}$. $100 \mu \mathrm{L}$ of 3-APES (3-aminopropyltriethoxysilane, Sigma-Aldrich, St. Louis, MO) is added onto the surface for $5 \mathrm{~min}$ and then $100 \mu \mathrm{L}$ of distilled water is added for $10 \mathrm{~min}$. The glass cover slip is thoroughly rinsed with ultrapure water to wash away any remaining 3-APES solution. Then, $100 \mu \mathrm{L}$ of $0.5 \%$ of glutaraldehyde (Sigma-Aldrich) in water ( $\mathrm{vol} / \mathrm{vol}$ ) is added onto the cover slip for $30 \mathrm{~min}$. The glass cover slip is subsequently rinsed with water. Polyacrylamide gel solutions are prepared using a $40 \% \mathrm{w} / \mathrm{v}$ acrylamide stock solution (BioRad, CA, USA) and a $2 \% \mathrm{w} / \mathrm{v}$ bisacrylamide stock solution (BioRad, CA, USA). PAA gel solutions are prepared with acrylamide and bis-acrylamide at final volume concentrations of $10 \% \mathrm{w} / \mathrm{v}$ and between 0.03 and $0.15 \% \mathrm{w} / \mathrm{v}$ respectively for gels with rigidities between 1 and $16.7 \mathrm{kPa}$. To polymerize the solution, $1 \mu \mathrm{L}$ TEMED (FisherBiotech) and $10 \mu \mathrm{L}$ of $10 \%$ ammonium persulfate are added with the appropriate amount of water to yield a final volume of $1 \mathrm{~mL}$. A fixed volume of $20 \mu \mathrm{L}$ of the polyacrylamide solution is immediately pipetted onto the center of the $25 \mathrm{~mm}$ diameter glass coverslip. A second untreated $25 \mathrm{~mm}$-diameter coverslip is then carefully placed on top of the 
polyacrylamide solution. Polymerization is completed in about $30 \mathrm{~min}$ and the top cover slip is slowly peeled off. The resultant gel exhibited a nearly ideal elastic behavior. A heterobifunctional crosslinker, sulfo-SANPAH (sulfosuccinimidyl 6-(4'-azido-2'-nitrophenylamino) hexanoate, Pierce Biotechnology, USA), is used to crosslink fibronectin molecules onto the surface of the gel. $500 \mu \mathrm{L}$ of a HEPES solution $(\mathrm{pH}=8.5)$ containing sulfo-SANPAH with a mass concentration of $0.2 \mathrm{mg} \mathrm{mL}^{-1}$ and EDC (1-ethyl-3[3-dimethylaminopropyl]carbodiimide hydrochloride; SigmaAldrich) with a mass concentration of $2 \mathrm{mg} \mathrm{mL}^{-1}$ is pipetted onto the gel surface. The polyacrylamide gel is then placed $10 \mathrm{~cm}$ below an ultraviolet lamp for $10 \mathrm{~min}$. We then washed the gel with PBS at $\mathrm{pH}$ 8.5. After the PBS solution is aspirated, $100 \mu \mathrm{L}$ of a $0.1 \mathrm{mg} \mathrm{mL}^{-1}$ fibronectin solution is pipetted on top of the polyacrylamide gel. After $45 \mathrm{~min}$, the gel is rinsed with PBS at $\mathrm{pH} 8.5$ for $10 \mathrm{~min}$. The cover slip with the attached polyacrylamide gel is placed at the bottom of a chamber for the experiment. The polyacrylamide gels fabricated in this manner were determined to be about $40 \mu \mathrm{m}$ thick immediately after formation and $80 \mu \mathrm{m}$ after swelling for 2 hours once the cell medium is added. All the substrates are coated with fibronectin. Thus, the elastic modulus $E$ is varied while a constant chemical environment is maintained.

\section{Microscopy and image analysis}

Aggregates are placed on the substrate at the bottom of a cylindrical experimental chamber filled with a $\mathrm{CO}_{2}$-equilibrated culture medium maintained at $37{ }^{\circ} \mathrm{C}$ using a heating cube system. To prevent evaporation, the open surface was sealed with mineral oil. The cell aggregate spreading was observed from below. Bright-field images were taken using a NIKON confocal microscope equipped with a $10 \times$ air objective. Bright field images are recorded every 5, 10 or 15 minutes with a CCD camera (Luca-R, Andor) using NIS-Elements software. Images were exported in the TIFF format and visualized using the ImageJ software package v.1.46r (National Institutes of Health, Bethesda, MD).

The presence of a precursor film is the signature of a spreading coefficient $S>0$. The spreading dynamics is characterized by measuring the contact and the precursor film areas by tracing the contours of the spreading aggregate, using ImageJ software and taking the enclosed area. The wound-healing experiments and the imaging of the densifying two-dimensional monolayer were performed under an automated phase contrast inverted microscope (Olympus IX71) with a $10 \times$ objective equipped with temperature, humidity and $\mathrm{CO}_{2}$ regulations (Life Imaging Services). The displacements of the sample and image acquisition with a CCD camera (EZ CoolSnap, Roper) were controlled through Metamorph (Universal Imaging) software. The typical delay between two successive images of the same field was $10 \mathrm{~min}$.

In addition to visualization, most of the image processing was performed using the ImageJ public domain software. ${ }^{22}$ The orientation field was obtained by computing the local structure tensor with ImageJ's plugin OrientationJ. ${ }^{23}$ The velocity field in the spreading film was mapped by particle image velocimetry
(PIV) analysis. ${ }^{24}$ Stacks of images were analyzed with a custom PIV algorithm based on the MatPIV software package for Matlab. ${ }^{25}$ The time between successive analyzed images was $10 \mathrm{~min}$. The window size was set to 32 pixels $(\sim 24 \mu \mathrm{m})$ and the overlap to 0.5 .

Live two-photon imaging was carried out on an inverted SP8 microscope (Leica), coupled with a femtosecond laser (Coherent) at a $980 \mathrm{~nm}$ excitation wavelength using a $40 \times 1.3$ N.A. oil immersion objective. The microscope was equipped with a top stage incubator (Okolab). The 3D and time datasets were acquired once every $5 \mathrm{~min}$ with a voxel size of $0.52 \times 0.52 \times 0.52 \mu \mathrm{m}$. The obtained datasets were visualized using Imaris (Bitplane; www.bitplane.com/imaris/ imaris) and Fiji ${ }^{26}$ software (http://fiji.sc/Fiji).

\section{Immunofluorescence staining of cell's aggregates and confocal microscopy imaging}

Aggregates of CT26 life-act GFP (incubated 3 days) are randomly deposited on the fibronectin-coated polyacrylamide gels, in a magnetic imaging chamber (Chamlide CMB, CM-B25-1) filled with the $\mathrm{CO} 2$-equilibrated culture medium. After lifetime image acquisition of aggregate spreading, the cell's aggregates are fixed and stained using the following protocol. Cells are fixed in 4\% paraformaldehyde (PFA)-PBS for $15 \mathrm{~min}$ and then washed with PBS. Cells are permeabilized and blocked with a BSATriton solution (1\% BSA, $0.1 \%$ Triton X-100 in PBS) on ice for $15 \mathrm{~min}$ and then rinsed with PBS solution for 5 min (3 times). A primary antibody solution (Anti-Laminin produced in rabbit, Sigma Aldrich) is added to the aggregates over night at $4{ }^{\circ} \mathrm{C}$ afterwards the chamber is rinsed with PBS for 5 min ( 3 times). Then a secondary antibody (goat anti rabbit IgG Alexa 633, Thermo Fisher Scientific) solution is added to the aggregates for $1 \mathrm{~h}$ and rinsed with PBS. Finally, the chamber is rinsed with PBS for 15 min ( 3 times). After staining, we observed the aggregates using an inverted confocal microscope (TCS SP5, Leica Microsystems) equipped with a $10 \times 0.30 \mathrm{NA}$ objective. Images are exported from the instrument software in the TIFF format and visualized using the ImageJ software package v.1.46r (National Institutes of Health, Bethesda, MD).

\section{Results and discussion}

We observe the formation of a precursor film on glass and PAA gels of elastic modulus ranging from $70 \mathrm{GPa}$ to $1 \mathrm{kPa}$. For each substrate rigidity, we characterize (i) the dynamics of spreading from the measurement of the spread area $A$ of the precursor film versus time shown in Fig. 1, (ii) the state of the film (liquid versus gas), (iii) the flow field in the spreading monolayer using particle image velocimetry (PIV) and finally (iv) the orientation of the cells. To determine if the cellular flows are purely radial such as for simple liquids or more disorganized, we defined and measured an order parameter $Q_{\mathrm{v}}=V_{\mathrm{r}} / V$, where $V_{\mathrm{r}}$ is the radial coordinate of the velocity field in polar coordinates and $V$ is the velocity amplitude $\left(\left\langle Q_{\mathrm{v}}\right\rangle=1\right.$ means that all the cells move radially outwards and $\left\langle Q_{\mathrm{v}}=0\right\rangle$ means that the cells move randomly). The cell orientation field is quantified by a nematic 


\section{Rigidity $\mathrm{E}$}

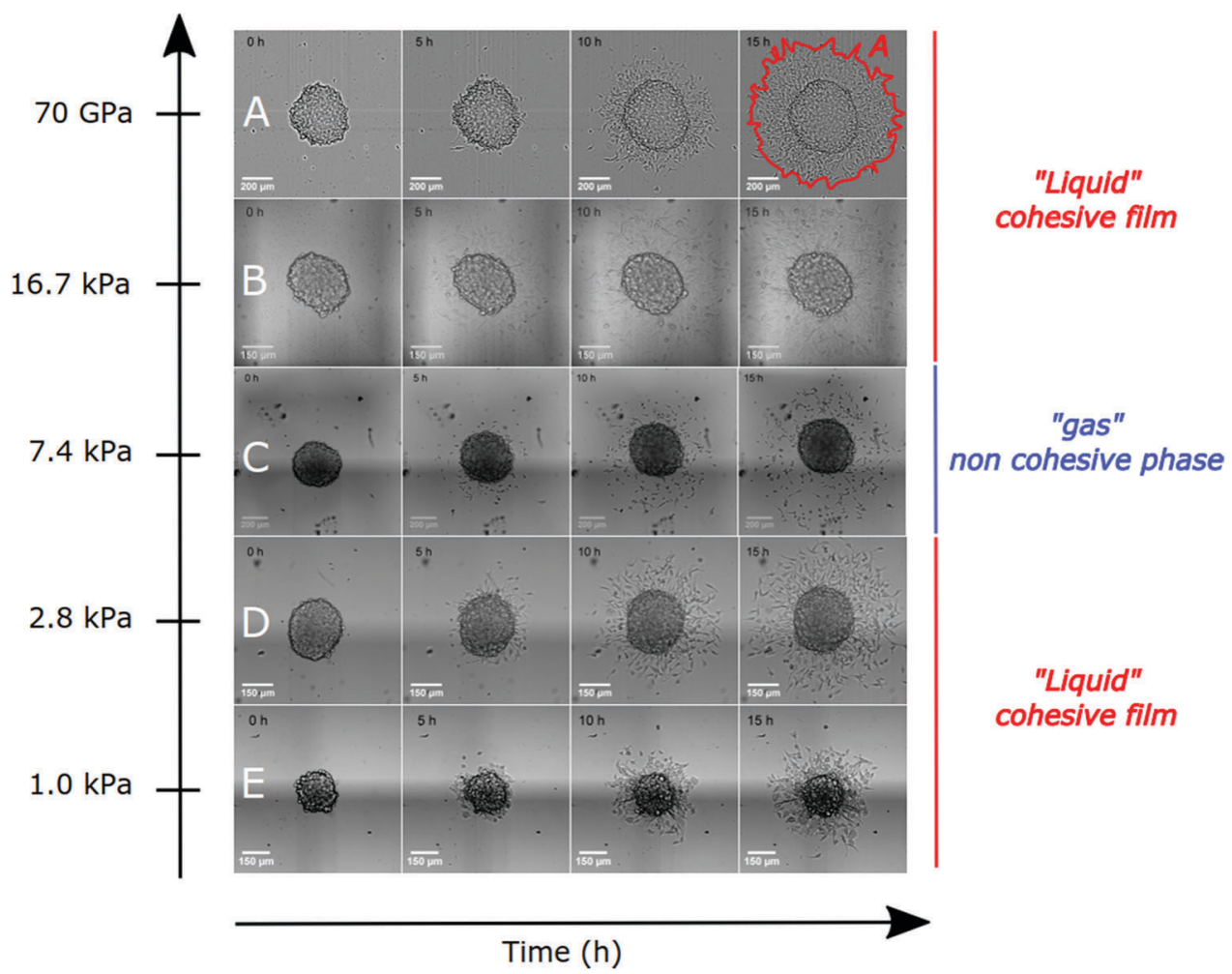

Fig. 1 Spreading of a CT26 aggregate on a spectrum of rigid to soft substrates coated with fibronectin. (A) Glass cover slip ( $E=70 \mathrm{GPa}$ ), (B) PAA gel $(E=16.7 \mathrm{kPa})$, (C) PAA gel $(E=7.4 \mathrm{kPa})$, (D) PAA gel $(E=2.8 \mathrm{kPa})$, and (E) PAA gel $(E=1 \mathrm{kPa})$. Scale bars and spreading times (in hours) are indicated on each image. Contact area $A$ is defined by the area enclosed by the red color.

order $Q$. We use the following scalar apolar order parameter in the spirit of the Landau-de Gennes theory of liquid crystals, ${ }^{27}$ $Q=\left\langle\cos \left(2 \theta_{\text {cell }}\right)\right\rangle$, where $\theta_{\text {cell }}$ is the angle between the cell long axis and the radial direction. In the limiting cases, $Q=0$ for a completely disordered state and $Q=1$ for a perfect nematic order where all the cells align radially.

\section{A. Observation of aggregate spreading versus substrate rigidity}

On rigid substrates (Fig. 1A, B and ESI, $\dagger$ Movies 1, 2), the precursor film is cohesive: all the cells within the monolayer have cell/cell contacts. Shortly after plating the aggregate, elongated isolated cells with thin protrusions spread outward from the aggregate. At longer times ( $t \geq 10 \mathrm{~h}$ ), collective motion occurs. The cell flow is radial. Moreover, the cells are oriented in the direction of the flow and they form a nematic corona characterized by a length $L_{\mathrm{N}}$ that increases with time.

On semi-rigid substrates (10.6 to $7.4 \mathrm{kPa}$, Fig. 1C), as the rigidity decreases, the precursor film appears less cohesive, leading to a liquid-gas transition. At $10.6 \mathrm{kPa}$ (ESI, $\uparrow$ Movie 3) the flow field is disordered and at $7.4 \mathrm{kPa}$ (Fig. 1C and ESI, $\uparrow$ Movie 4) cells escape individually from the aggregate. The precursor film does not form a continuous monolayer: cells are scattered and form a gas state that spreads much faster.

On softer substrates ( 2.8 and $1 \mathrm{kPa}$ ) the precursor film becomes cohesive again and we also observe a nematic corona Fig. 1D (ESI, $\dagger$ Movie 5) and Fig. 1E (ESI, $\uparrow$ Movie 6). The cells form cell-cell adhesion, the monlayer is continuous and is in a liquid state. The apparition of a reentrant liquid-gas transition is an unexpected and new result. Reentrant phase transition has been first described in the field of thermotropic liquid crystals, where a nematicsmectic-nematic phase transition has been observed under cooling. ${ }^{28}$ Here, we attribute this reentrant liquid phase to the slowdown of the cell spreading on soft substrates. The velocity is strongly reduced (Fig. 2) and during this slow process more ECMs were secreted by the cells. ECM accumulation can increase cell/cell adhesion leading to a cohesive liquid state. We clearly see (Fig. S2, $\mathrm{ESI}+$ ) that the cells secrete laminin, one protein of the ECM. Foty et $a .^{29}$ studied the influence of ECM accumulation on cell migration and cell/cell adhesion. They showed that drugs that can activate a cell's ability to assemble fibronectin into a complex fibrous matrix limit the propagation of very aggressive glioblastoma cells by increasing cell-cell adhesion. To test this hypothesis of ECM accumulation leading to reduce cell migration dynamics, we have compared the dynamics of spreading of aggregates that have been incubated for either 3 or 6 days. As shown in Fig. 2, the velocity of spreading is reduced by a factor of order 2 when the cells have been incubated longer, suggesting that ECM proteins accumulate in the aggregate over time, leading to a slower spreading.

\section{B. Dynamics of spreading}

We plot the spread area, $A$, of the precursor film versus time for all substrates of different rigidities. The dynamics of spreading 
A

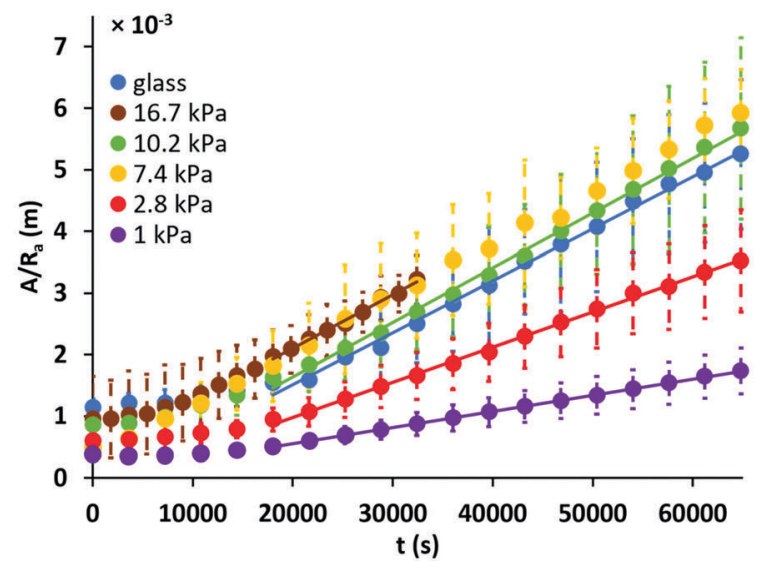

B

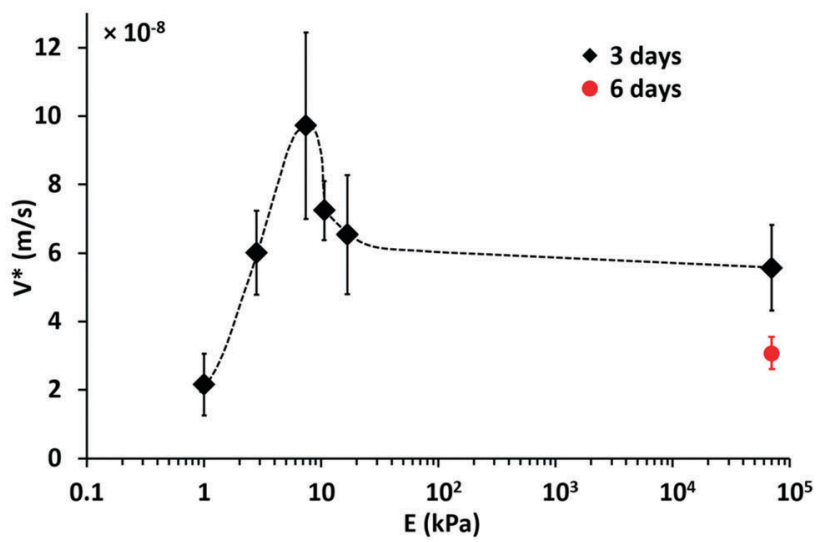

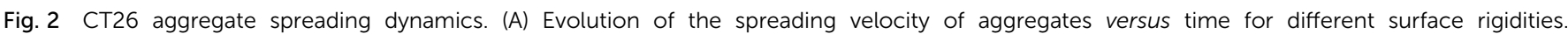

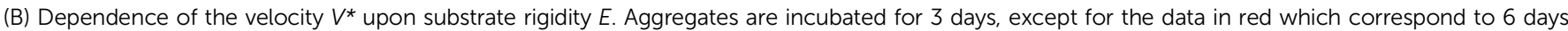

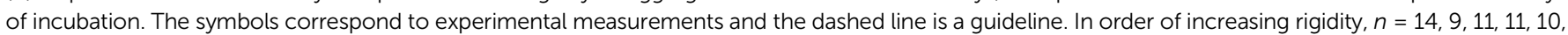
and 11 for the 3 day experiments and $n=15$ for the 6 day experiments.

is modeled by a balance between the active driving forces $(S$, the spreading parameter) due to motile cells at the film periphery ${ }^{30,31}$ and the viscous dissipation associated with two following types of cellular flow: ${ }^{7}$ (i) the permeation corresponding to the passage of cells from the aggregate into the film and (ii) the slippage as the film expands. Both flows will give rise to a diffusive spreading law, with either a diffusion coefficient $D$ that is proportional to the radius $R_{\mathrm{a}}$ of the aggregate if the dissipation due to the permeation is dominant or a diffusion coefficient $D$ that is almost independent of $R_{\mathrm{a}}\left(D \sim \ln \left(R_{\mathrm{a}}\right)\right)$ if the sliding is dominant. We identified that for CT26 the diffusion coefficient on glass and PAA substrates fulfils the first criteria and is proportional to $R_{\mathrm{a}}$, demonstrating that the dissipation is dominated by the permeation of the cells that flow from the $3 \mathrm{D}$ aggregate into the $2 \mathrm{D}$ monolayer. ${ }^{7}$

A diffusion coefficient proportional to $R_{\mathrm{a}}$ can be modeled by the following dynamical law for the contact area $A$ versus time $t$ :

$$
A=R_{\mathrm{a}} V^{*} t=D t
$$

where $V^{*}=2 \pi \frac{S}{\eta}$ is a typical spreading velocity and $R_{\mathrm{a}}$ is the aggregate radius (see also Fig. S1, ESI $\dagger$ ).

The spread area $A(t)$, shown in Fig. 2A, increases linearly in good agreement with the theoretical law in eqn (1). We therefore express the diffusion coefficient depending on the substrate rigidity as $D(E)=R_{\mathrm{a}} V^{*}$. To have an intrinsic parameter independent upon the size of the aggregate, we plot $\frac{D(E)}{R_{\mathrm{a}}}=V^{*}$ versus the elastic modulus (Fig. 2B).

We observe that $V^{*}$ reaches a maximum corresponding to an elastic modulus $E \sim 7 \mathrm{kPa}$, and decreases on ultra-soft substrates. For $E=1 \mathrm{kPa}$, the cohesive precursor film flows with a velocity $V^{*}=2 \times 10^{-8} \mathrm{~m} \mathrm{~s}^{-1}$, which is about five times slower than on a rigid substrate. We expect by extrapolation of $V^{*}(E)$ a wetting transition from complete to partial wetting to occur around $0.5 \mathrm{kPa}$. Overall, the spreading speed of CT26 cells on soft substrates is similar to the one obtained previously for S-180 cells that do not secrete ECM proteins. The main difference comes from the coherency of the flows at ultra-low rigidity $(E<2.8 \mathrm{kPa})$ and the re-entrant gas-liquid transition.

\section{Flow field}

We show in Fig. 3A the spreading of a cellular aggregate on a fibronectin coated glass cover slip from 10 to 30 hours. The cellular flow field measured using Particle Image Velocimetry (PIV) is shown in blue. Similarly, in Fig. 3B, we show spreading of aggregates at 20 hours on PAA substrates of increasing stiffness, and the corresponding flow fields in blue.

We quantify the coherency of the cellular velocity fields by measuring an order parameter map $Q_{\mathrm{v}}$, as defined in paragraph III.

Fig. 3C shows $Q_{v}$ for two aggregates spreading on glass and on a $7.4 \mathrm{kPa}$ substrate. We observe that when the precursor film is in a liquid state on the glass substrate the flow is radial $\left(Q_{\mathrm{v}} \sim 1\right.$ everywhere), while on the soft substrate $Q_{\mathrm{v}} \ll 1$, the film is in a gas state. Cells migrate individually in all directions as shown by the scattered distribution of the velocity field. The order parameter $Q_{\mathrm{v}}$ averaged over the entire precursor film versus the substrate elastic modulus (Fig. 3D) shows that the film is less cohesive (gas state) only at intermediate rigidities.

\section{Nematic order}

We characterize the nematic order, which appears during the spreading. We notice that CT26 cells do not have a nematic order when cultured as a $2 \mathrm{D}$ monolayer regardless of the cell concentration (Fig. S3, ESI $\dagger$ ). The nematic order during spreading is thus induced by the flows. Cells within the expanding layer on rigid substrates ( $E=70 \mathrm{GPa}$ and $16.4 \mathrm{kPa}$ ) exhibit membrane protrusion and rod like shapes. We observe a nematic order of the cells, which align parallel to the radial flows.

Fig. 4A shows the radial alignment of the cells spreading out on a glass substrate. To quantify the distance over which the 


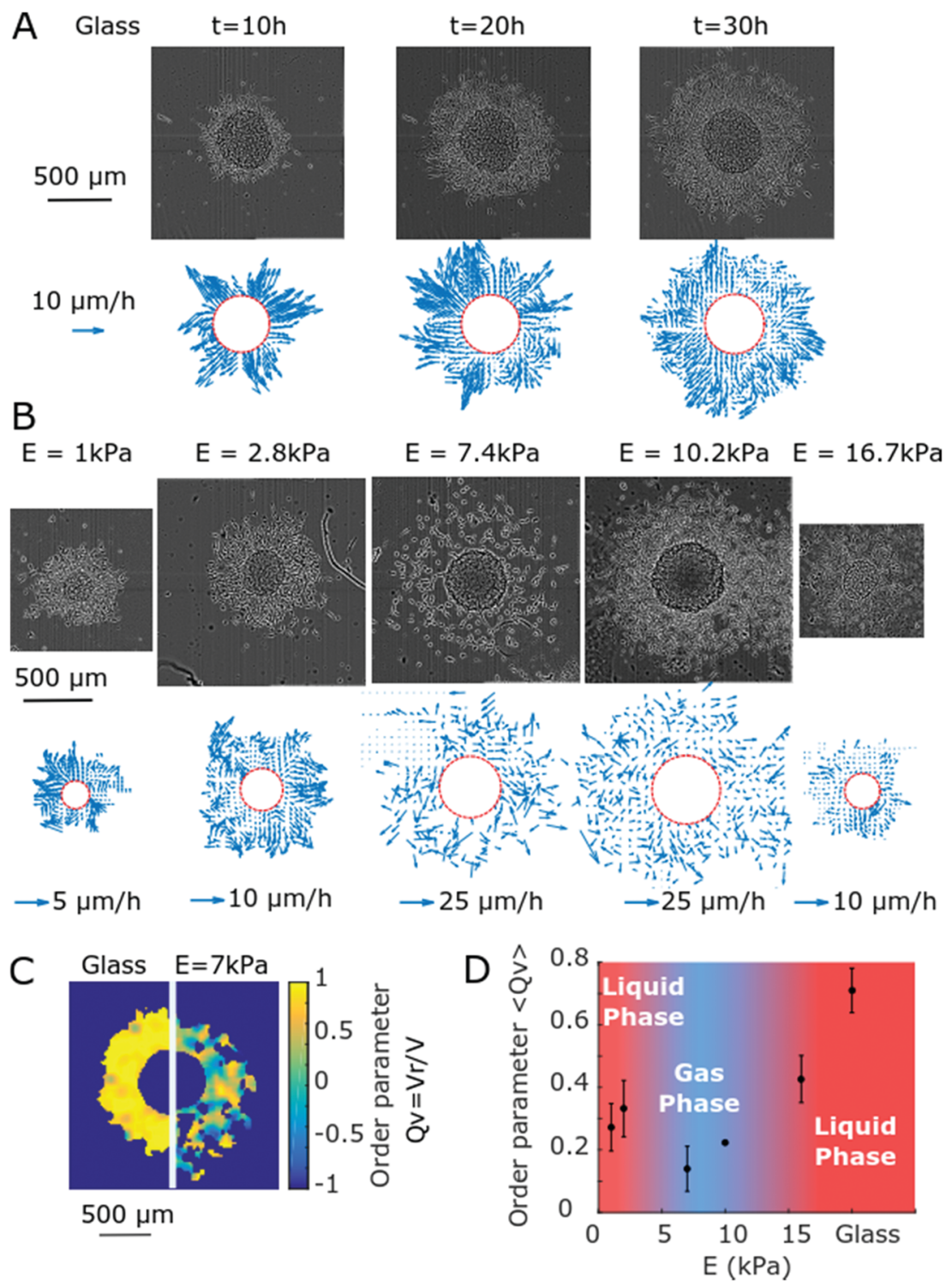

Fig. 3 Collective behavior of the cells in the spreading film. (A) Spreading of a CT26 aggregate on a fibronectin coated glass cover slip. Velocity maps in blue. (B) Spreading of CT26 aggregates on PAA substrates of increasing stiffness, $t=20 \mathrm{~h}$. (C) Heatmap of the radial order parameter $Q_{v}=V_{r} /|V|$ of the cell displacement on glass (left) and on a $7.4 \mathrm{kPa}$ PAA substrate (right). $V_{\mathrm{r}}$ is the radial velocity and $|\mathrm{V}|$ is the velocity amplitude. $Q_{\mathrm{v}}=1$ if all the cells move radially outwards and $Q_{v}=0$ if the cells move orthoradially, $t=20 \mathrm{~h}$. (D) Variation of the order parameter $Q_{v}$ averaged over the entire precursor film versus rigidity of the substrate. Large $\left\langle Q_{v}\right\rangle$ are associated with highly correlated radial movement of the cells (liquid phase) while low $\left\langle Q_{v}\right\rangle$ are associated with random uncorrelated movement of the cells (gas phase), $t=20 \mathrm{~h}$. The error bars are the standard deviation.

cells align, we radially average the apolar order parameter $Q=\left\langle\cos \left(2 \theta_{\text {cell }}\right)\right\rangle$, as defined in paragraph III. Fig. $4 \mathrm{D}$ shows the variation of the nematic order versus the distance $r$ to the center of the aggregate. We see that for the aggregate spreading on a glass substrate, the cells are aligned radially $(Q>0.5)$ in a corona of size $L_{\mathrm{N}}$ up to $160 \mu \mathrm{m}$ (about 10 cell length, see also Fig. S1, ESI $\dagger$ ). For aggregates spreading on soft substrates $(E=7.4 \mathrm{kPa}$ and $10.6 \mathrm{kPa})$, the cells are oriented randomly $(Q=0)$ and therefore $L_{\mathrm{N}}=0 \mu \mathrm{m}$. Fig. $4 \mathrm{E}$ shows the time evolution of the nematic length $L_{\mathrm{N}}$ on stiff substrate (both for glass and $E=16.7 \mathrm{kPa})$ and ultra-soft substrate $(E=1$ and $2.8 \mathrm{kPa})$. Fig. $4 \mathrm{G}$ summarizes the results as a phase diagram and Fig. $4 \mathrm{E}$ shows for comparison the time trajectories of $L_{\mathrm{N}}(t)$ and $R(t)-R_{\mathrm{a}}$, where $R(t)$ is the radius of the precursor film when the aggregates spread on a glass substrate.

To identify the origin of the nematic ordering we performed the following experiments. First, we culture CT26 cells as twodimensional monolayers on a fibronectin coated glass substrate. The cells do not exhibit any nematic alignment $(Q=0.19 \pm 0.09$, Fig. S3, ESI $\dagger$ ). Therefore steric cell-cell interactions are not enough to explain the nematic alignment of these cells, contrary to other cell types such as NIH fibroblasts, RPE1 epithelial cells, or C2C12 myoblasts. ${ }^{32,33}$ Secondly, we performed a control wound healing assay on a fibronectin coated glass substrate to identify if directed migration was enough to obtain the nematic alignment of the cells. Surprisingly, as the cells migrate to close 

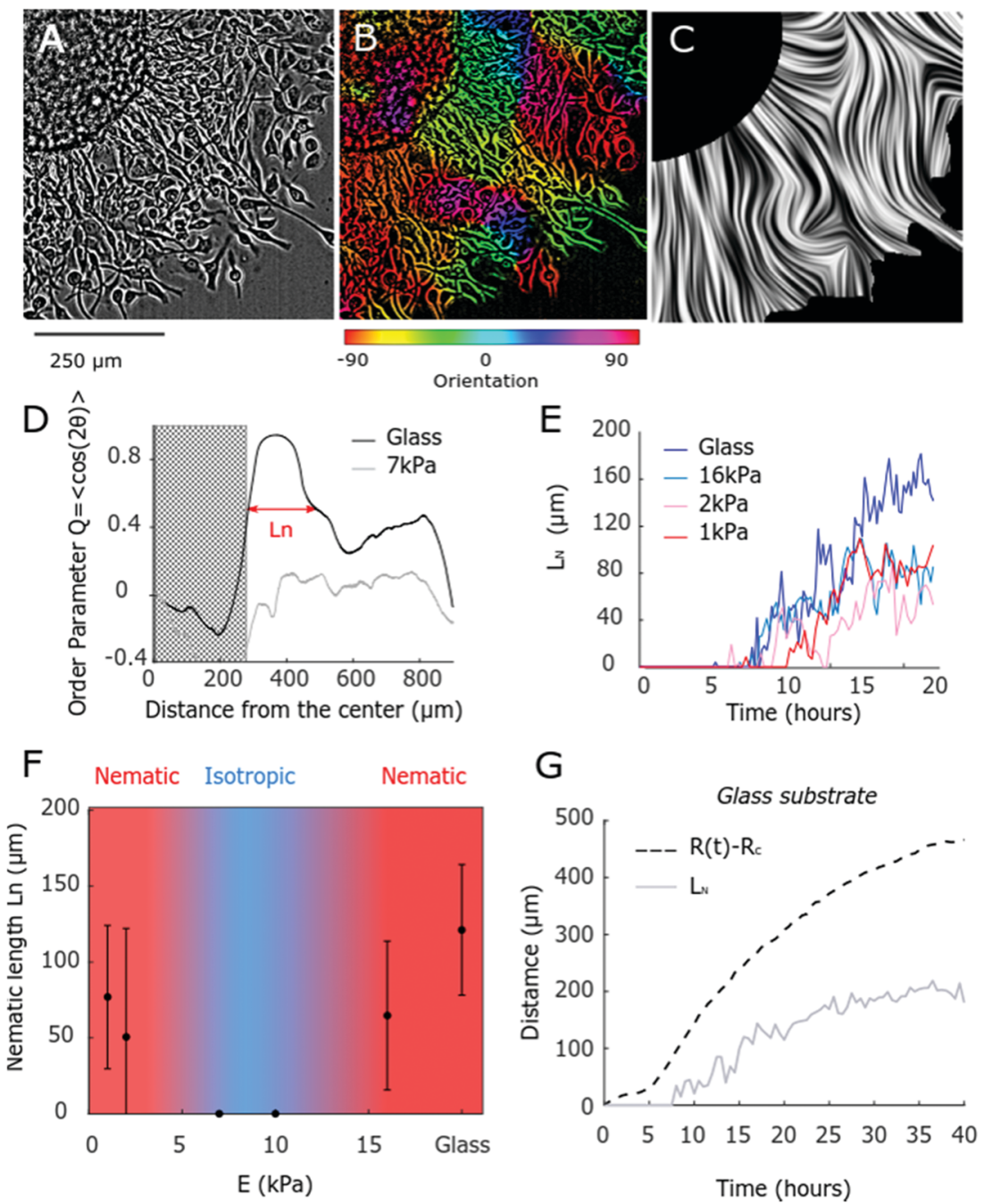

Fig. 4 Cell organization in the precursor film. (A) Bright field image of the precursor film of an aggregate spreading on glass at $t=20 \mathrm{~h}$. (B) The same image where cell orientation is color coded. (C) The same image where the orientation field is represented using the Line Integral Convolution technique. (D) Radial average of the order parameter $Q=\left\langle\cos \left(2 \theta_{\text {cell }}\right)\right\rangle$ where $\theta_{\text {cell }}$ is the angle between the cell long axis and the radial direction. The nematic length $L_{N}$ is defined as the length for which $Q$ is greater than 0.5. (E) Time evolution of the nematic length $L_{N}$ for aggregates spreading on glass and on PAA substrates $(E=16.7 \mathrm{kPa}, 2.8 \mathrm{kPa}$, and $1 \mathrm{kPa})$. For $E=10.6 \mathrm{kPa}$ and $E=7.4 \mathrm{kPa}$, the order parameter $Q$ is never greater than 0.5 and therefore $L_{\mathrm{N}}$ is constant and null. (F) Variation of the nematic length measured at $t=20 \mathrm{~h}$ for varying substrate rigidity. The first $\mathrm{N}-\mathrm{I}$ transition appear between 10.6 and $16.7 \mathrm{kPa}$ and the second I-N between 7.4 and $2.8 \mathrm{kPa}$. The error bars are the standard deviation. (G) Comparison of the time trajectory of $L_{\mathrm{N}}$ with $R(t)-R_{\mathrm{C}}$ when spreading on glass substrate.

the wound, we did not observe any nematic alignment (Fig. S4D and G, ESI $\dagger$ ) illustrating that uniform cell flows (Fig. S4F, ESI $\dagger$ ) are not enough to explain the nematic ordering either. We can therefore conclude that the nematic order is induced by the flow of the cells from the reservoir (3D aggregate) into the film. In this region, between the aggregate and the $2 \mathrm{D}$ monolayer, there is a strong converging flow. It has been shown both theoretically and experimentally that converging flows align rod-like bacteria, whereas diverging flows misalign them. ${ }^{34}$ This can explain the cell alignment near the aggregate-film contact line where the nematic corona is formed, and the progressive misalignment in the far field region where the velocity field diverges $(V(r)$ decreases as $1 / r$ at the aggregate-film contact line if we assume a constant density in the spreading film). No alignment has been observed with a mouse sarcoma cell line (S-180) transfected to express E-cadherin (Fig. S5, ESI $\dagger$ ). ${ }^{35}$ One difference between S-180 and CT26 cells is their mode of migration. CT26 cells move by the protrusion of filopodia and they align in the direction of the motion (ESI, $\dagger$ Movie S7). On the other hand, S-180 cells move by protrusion of lamellipodia and they are elongated in the direction perpendicular to the motion. We also observe that this nematic order appears only for relatively dense and cohesive monolayers (stiff and soft substrates) meaning that both flow alignment and density seem required to obtain a nematic phase.

\section{Conclusion}

In this paper, we have studied the spreading of CT26 cellular aggregates on glass (70 GPa) and hydrogels covered with 
fibronectin of rigidity ranging between 1 and $16.7 \mathrm{kPa}$. We have identified a novel rigidity dependent alignment of cells spreading from the 3D aggregate to a $2 \mathrm{D}$ monolayer. On glass and $16.7 \mathrm{kPa}$ PAA gel, cells appear elongated and locally aligned with their neighbors, forming highly ordered nematic domains aligned in the radial direction with a nematic order parameter $Q \cong 0.8$. It has been reported previously that spindle-shaped, weakly interacting, motile cells constitute an active out-equilibrium system that undergoes a phase transition from a low density disordered state to a highly ordered nematic state after confluence. ${ }^{32}$ Interestingly here CT26 cells cultured as a two-dimensional monolayer never order in a nematic phase whatever the density is. Moreover, we performed wound-healing experiments where the velocity profile is uniform and showed that cell orientation remains isotropic. We conclude that the observed nematic order could be induced by the flow pattern in our geometry. The nematic order which shows up at the periphery of the aggregate could be induced by the convergent flow of cells from the 3D aggregate into the film. Decreasing the elastic modulus of the matrix, we observe first a decrease of the cell density in the precursor film and a decrease of the cell collective alignment up to a complete gas phase where the precursor film is not cohesive and cells escape individually from the aggregate with an amoeboid migration type. ${ }^{36}$ The velocity of spreading reaches a maximum value for $E \sim 7.4 \mathrm{kPa}$. For smaller substrate rigidities, $(E \leq 2.8 \mathrm{kPa}$, ESI, $\dagger$ Movies 5-7) we find unexpected results. The cohesion of the film increases and cells align in a nematic corona at the contact line between the aggregate and the dense precursor film. At $2.8 \mathrm{kPa}$ the cohesion of the film is reestablished and increases for even lower rigidities. We investigated the case of reentrant gasliquid and nematic transitions. The reentrant liquid phase could be attributed to the role of the cellular matrix secreted by CT26 cells when the spreading velocity becomes extremely small as shown by Foty et $a l^{13}$

The observation of a reentrant liquid-gas-liquid phase transition while decreasing the elastic modulus is an important and interesting result. It shows that the EMT transition can be reversed by the rigidity of the substrate for cells secreting ECM proteins. Our finding that cell escape can be hindered on soft substrates for cells secreting ECM proteins may have applications in cancer research and drug formulation. We also observed a reentrant nematic phase where the control parameter is the cell/substrate interaction that can be tuned by the substrate rigidity. In liquid crystals, a nematic phase reappears after passing through a smectic-A phase by lowering the temperature. In our case, the liquid phase occurs for both rigid (high $E$ ) and ultra-soft (low $E$ ) substrates. On rigid substrates cell/substrate adhesions $\left(W_{\mathrm{CS}}\right)$ are high and they decrease on soft substrates. ${ }^{12}$ Because of the cross-talks between the cadherin and the integrin, the cell/cell adhesion $W_{\mathrm{CC}}$ decreases with $W_{\mathrm{CS}}$. This cross-talk explains the first liquid/gas transition observed when decreasing the substrate rigidity, as demonstrated previously for S-180 cells that do not secrete ECMs. The reentrant liquid phase indicates that cell/cell adhesion increased. We attribute the non-monotonic behavior of $W_{\mathrm{CC}}$ that increases again on an ultra-soft substrate to the accumulation of the ECM, which glues the cells together when the spreading of the precursor film becomes very slow. The characteristic secretion times are of order of a few hours while the spreading velocity is on the order of $1 \mu \mathrm{m} \mathrm{h}^{-1}$, i.e. one cell's body length for 5-10 hours. We also observe on ultra-soft substrates a reentrant nematic liquid crystal phase as the cell density in the film increases that we attribute, as during the spreading on a rigid substrate, to the convergent flows from the aggregate into the $2 \mathrm{D}$ monolayer.

\section{Conflicts of interest}

There are no conflicts to declare.

\section{Acknowledgements}

We thank Basile Gurchenkov (IGBMC, Strasbourg, France) for the 2-photon experiments and Pascal Silberzan (Institut Curie, UMR 168, Paris, France) for useful discussions. The BiologyInspired Physics at Mesoscales group (BIPM) and the Soft Surfaces group are members of the Labex CelTisPhyBio. The BIPM is "Equipe labellisée Ligue Nationale Contre le Cancer" and member of the IPGG.

\section{References}

1 N. Wang, J. P. Butler and D. E. Ingber, Science, 1993, 260, 1124-1127.

2 P. R. Girard and R. M. Nerem, J. Cell. Physiol., 1995, 163, 179-193.

3 S. R. Peyton and A. J. Putnam, J. Cell. Physiol., 2005, 204, 198-209.

4 P. Friedl and D. Gilmour, Nat. Rev. Mol. Cell Biol., 2009, 10, 445-457.

5 P. Friedl, J. Locker, E. Sahai and J. E. Segall, Nat. Cell Biol., 2012, 14, 777-783.

6 F. Montel, M. Delarue, J. Elgeti, L. Malaquin, M. Basan, T. Risler, B. Cabane, D. Vignjevic, J. Prost, G. Cappello and J.-F. Joanny, Phys. Rev. Lett., 2011, 107, 188102.

7 G. Beaune, T. V. Stirbat, N. Khalifat, O. Cochet-Escartin, S. Garcia, V. V. Gurchenkov, M. P. Murrell, S. Dufour, D. Cuvelier and F. Brochard-Wyart, Proc. Natl. Acad. Sci. U. S. A., 2014, 111, 8055-8060.

8 M. S. Steinberg, Science, 1963, 141, 401-408.

9 D. Gonzalez-Rodriguez, K. Guevorkian, S. Douezan and F. Brochard-Wyart, Science, 2012, 338, 910-917.

10 S. Douezan, K. Guevorkian, R. Naouar, S. Dufour, D. Cuvelier and F. Brochard-Wyart, Proc. Natl. Acad. Sci. U. S. A., 2011, 108, 7315-7320.

11 S. Douezan, J. Dumond and F. Brochard-Wyart, Soft Matter, 2012, 8, 4578.

12 A. Zemel, F. Rehfeldt, A. E. X. Brown, D. E. Discher and S. A. Safran, Nat. Phys., 2010, 6, 468-473.

13 P. L. Ryan, R. A. Foty, J. Kohn and M. S. Steinberg, Proc. Natl. Acad. Sci. U. S. A., 2001, 98, 4323-4327.

14 F. Broders-Bondon, P. Paul-Gilloteaux, C. Carlier, G. L. Radice and S. Dufour, Dev. Biol., 2012, 364, 178-191. 
15 M. A. Nieto, R. Y.-J. Huang, R. A. Jackson and J. P. Thiery, Cell, 2016, 166, 21-45.

16 C. Martinez-Rico, F. Pincet, J.-P. Thiery and S. Dufour, J. Cell Sci., 2010, 123, 712-722.

17 K. Guevorkian, M.-J. Colbert, M. Durth, S. Dufour and F. Brochard-Wyart, Phys. Rev. Lett., 2010, 104, 218101.

18 N. Belot, S. Rorive, I. Doyen, F. Lefranc, E. Bruyneel, R. Dedecker, S. Micik, J. Brotchi, C. Decaestecker, I. Salmon, R. Kiss and I. Camby, Glia, 2001, 36, 375-390.

19 K. Alessandri, B. R. Sarangi, V. V. Gurchenkov, B. Sinha, T. R. Kießling, L. Fetler, F. Rico, S. Scheuring, C. Lamaze, A. Simon, S. Geraldo, D. Vignjevic, H. Doméjean, L. Rolland, A. Funfak, J. Bibette, N. Bremond and P. Nassoy, Proc. Natl. Acad. Sci. U. S. A., 2013, 110, 14843-14848.

20 P. Marmottant, A. Mgharbel, J. Käfer, B. Audren, J.-P. Rieu, J.-C. Vial, B. van der Sanden, A. F. M. Marée, F. Graner and H. Delanoë-Ayari, Proc. Natl. Acad. Sci. U. S. A., 2009, 106, 17271-17275.

21 Y.-L. Wang and R. J. Pelham Jr., Preparation of a flexible, porous polyacrylamide substrate for mechanical studies of cultured cells, Academic Press Inc., 525 B Street, Suite 1900, San Diego, CA 92101-4495, USA, 1998, vol. 298.

22 W. S. Rasband, ImageJ, U.S. National Institutes of Health, Bethesda, Maryland, USA, 1997-2016, https:/imagej.nih.gov/ij/.

23 R. Rezakhaniha, A. Agianniotis, J. T. C. Schrauwen, A. Griffa, D. Sage, C. V. C. Bouten, F. N. van de Vosse, M. Unser and N. Stergiopulos, Biomech. Model. Mechanobiol., 2012, 11, 461-473.

24 L. Petitjean, M. Reffay, E. Grasland-Mongrain, M. Poujade, B. Ladoux, A. Buguin and P. Silberzan, Biophys. J., 2010, 98, 1790-1800.
25 M. Deforet, M. C. Parrini, L. Petitjean, M. Biondini, A. Buguin, J. Camonis and P. Silberzan, Nat. Methods, 2012, 9, 1081-1083.

26 J. Schindelin, I. Arganda-Carreras, E. Frise, V. Kaynig, M. Longair, T. Pietzsch, S. Preibisch, C. Rueden, S. Saalfeld, B. Schmid, J.-Y. Tinevez, D. J. White, V. Hartenstein, K. Eliceiri, P. Tomancak and A. Cardona, Nat. Methods, 2012, 9, 676-682.

27 P. G. de Gennes and J. Prost, The physics of liquid crystals, Oxford University Press, Oxford, 2nd edn, 2003.

28 P. E. Cladis, Phys. Rev. Lett., 1975, 35, 48-51.

29 J. Sabari, D. Lax, D. Connors, I. Brotman, E. Mindrebo, C. Butler, I. Entersz, D. Jia and R. A. Foty, PLoS One, 2011, 6, e24810.

30 X. Trepat, M. R. Wasserman, T. E. Angelini, E. Millet, D. A. Weitz, J. P. Butler and J. J. Fredberg, Nat. Phys., 2009, 5, 426-430.

31 A. Saez, E. Anon, M. Ghibaudo, O. du Roure, J.-M. Di Meglio, P. Hersen, P. Silberzan, A. Buguin and B. Ladoux, J. Phys.: Condens. Matter, 2010, 22, 194119.

32 G. Duclos, S. Garcia, H. G. Yevick and P. Silberzan, Soft Matter, 2014, 10, 2346-2353.

33 G. Duclos, C. Erlenkämper, J.-F. Joanny and P. Silberzan, Nat. Phys., 2016, 13, 58-62.

34 E. Katz, A. L. Yarin, W. Salalha and E. Zussman, J. Appl. Phys., 2006, 100, 34313.

35 Y.-S. Chu, W. A. Thomas, O. Eder, F. Pincet, E. Perez, J. P. Thiery and S. Dufour, J. Cell Biol., 2004, 167, 1183-1194.

36 Y.-J. Liu, M. Le Berre, F. Lautenschlaeger, P. Maiuri, A. Callan-Jones, M. Heuzé, T. Takaki, R. Voituriez and M. Piel, Cell, 2015, 160, 659-672. 